Acta vet. scand. 1984, 25, 346-351.

From the Department of Surgery, College of Veterinary Medicine, Helsinki and the Department of Ophthalmology, University of Helsinki, Finland.

\title{
FLUORESCEIN ANGIOGRAPHY OF THE CANINE OCULAR FUNDUS IN KETAMINE-XYLAZINE ANESTHESIA
}

\author{
By \\ B. Kommonen and L. Koskinen
}

KOMMONEN, B. and L. KOSKINEN: Fluorescein angiography of the canine ocular fundus in ketamine-xylazine anesthesia. Acta vet. scand. 1984, 25, 346-351. - Fluorescein fundus angiography was performed on 30 dogs with no pathologic fundus findings using a Canon $\mathrm{CF} 60 \mathrm{Z}$ wide angle camera. In serial photography of the dye passage 4 different circulatory phases could be distinguished: choroidal, retinal anteriolar, capillary and venous phase. The dye passage in the retinal vessels was described in detail. For aesthesia the combination of ketamine and xylazine proved to be suitable.

staining; retinal blood vessels; dog.

Fluorescein angiography has been used for several years to study retinal circulation before being used in clinical work. Bellhorn (1973) described fluorescein fundus photography in animals. Gelatt et al. (1976) compared restraint, different types of films and various doses of fluorescein in the dog.

The fluorescein injected intravenously appears in the eye in a few seconds in the dog, as in most animals. Sodium fluorescein is excited by blue light of $490 \mathrm{~nm}$ and after excitation emits light at a wave-length of $520 \mathrm{~nm}$. By using a blue filter over the strobe light and a yellow filter in front of the film it is possible to observe the dye as it passes through the choroid and retinal vessels. Defects in the blood retinal barrier and choroid-retinal barriers are seen as dye leaks into the retina and vitreous (Gelatt 1981).

In this study fluorescein angiography of ophthalmologically healthy dogs was performed using a photographic unit which 
gives a wide angle $\left(60^{\circ}\right)$ wiew of the fundus. Previous reports have not described the passage of the dye in the retinal capillaris in the dog in detail.

\section{MATERIAL AND METHODS}

A total of 30 clinically healthy dogs of both sexes and different breeds (Table 1), age ranging from 6 months to 8 years, were examined at the Department of Ophthalmology, University of Helsinki.

T a b l e 1. Breeds and numbers of the dogs examined.

\begin{tabular}{lc}
\hline Breeds & Number of dogs \\
\hline Toy Poodle & 14 \\
Miniature Poodle & 5 \\
Giant Poodle & 5 \\
Beagle & 4 \\
Labrador Retriever & 1 \\
Chesapeake Bay Retriever & 1 \\
\hline
\end{tabular}

At the general examination none of the dogs showed signs of an ophthalmological or general disease. Prior to angiography the dogs were examined by direct and indirect ophthalmoscopy. Slit lamp biomicroscopy was performed in order to detect possible lens and vitreous opacities. None of the dogs had pathologic findings. The dogs were electroretinographically tested and were all found to be normal.

For mydriasis cyclopentolate $5 \mathrm{mg} / \mathrm{ml}$ (Oftan Syklo ${ }^{\circledR}$, Star) and tropicamide $5 \mathrm{mg} / \mathrm{ml}$ (Oftan Tropicamid $\circledast$, Star) eyedrops were given approximately 20 min prior to photography.

After an overnight fast the dogs were anesthesized using a combination of ketamine hydrochloride $9 \mathrm{mg} / \mathrm{kg}$ (Ketalar ${ }^{\circledR} 5$ $\mathrm{mg} / \mathrm{ml}$, Parke Davis) and xylazine hydrochloride $1.4 \mathrm{mg} / \mathrm{kg}$ (Rompun vet. $\circledast 20 \mathrm{mg} / \mathrm{ml}$, Bayer) given intramuscularly in the biceps femoris muscle or slowly intravenously in the cephalic vein. Within $10 \mathrm{~min}$ of administration of the anesthetics the dogs were placed in lateral recumbency on their right side on a table in front of a Canon $\mathrm{CF} Z$ fundus camera.

The camera was focused on the fundus and control photographs on Kodak Tri-X pan 400 ASA film or Kodak ektachrome 64 film were taken. Sodium fluorescein $10 \%$ aquaeous solution 
(Fluorescite $\AA 10 \%$ inject, Alcon) was rapidly injected in the left cephalic vein using a dosage of $12 \mathrm{mg} / \mathrm{kg}$. Photography was started prior to appearance of the dye in the retinal arterioles, and was continued at $1.0 \mathrm{~s}$ intervals for $15-30 \mathrm{~s}$. During anesthesia the cornea was frequently moistened with $0.9 \%$ saline solution.

Angiography pictures were taken on Kodak Tri-X pan 400 ASA film. The camera had build-in excitatory and barrier filters so no extra filters were needed during angiograhy. For the control photographs a Kodak Wratten 58 filter was used. The black and white films were developed in Agfa Refinal ${ }^{\circledR}$ according to the method used at the Department of Ophthalmology. The developing time was $7 \mathrm{~min}$ at $20^{\circ} \mathrm{C}$.

\section{RESULTS}

During the initial phase of angiography some of the dogs showed autofluorescence of the tapetum. The dye appeared approximately at the same time in the choroidal circulation as in the $10-20$ cilioretinal arterioles. The choroidal filling was first seen as a patchy fluorescence in the nontapetal region (Fig. 1).

In the early arteriolar phase the $10-20$ cilioretinal vessels emerging from the optic disc periphery seemed to fill almost simultaneously Fig. 1). Differences in the filling velocity of individual arterioles were noted; the filling of those in the tapetal and nontapetal parts of the fundus was simultaneous.

The dye first appeared in the retinal capillaries surrounding the arteriolar branches near the optic disc region (Fig. 3). The capillaries were poorly visualized in the early venous phase (Fig. 2 ). The fluorescence of the capillary bed was most obvious during the mid-venous phase in the majority of the dogs (Figs. 3 and 4 ). A narrow capillary free zone along the arterioles was observed (Figs. 4 and 8 ). In the periphery the capillary bed clearly became less dense (Figs. 4 and 7 ), and close to the ora serrata the network was totally lacking. The capillaries were not visualized in the tapetal area because of autofluorescence (Fig. 4 ). The background fluorescence reached its maximum approximately at the same time as the capillary fluorescence was seen in the mid-periphery of the fundus (Fig. 4).

The first branches to fill during the venous phase were the veins of the area centralis region. The dye laminated against the 
B. Kommonen and L. Koskinen: Fluorescein Angiography of the canine ocular fundus in ketamine-xylazine anesthesia.
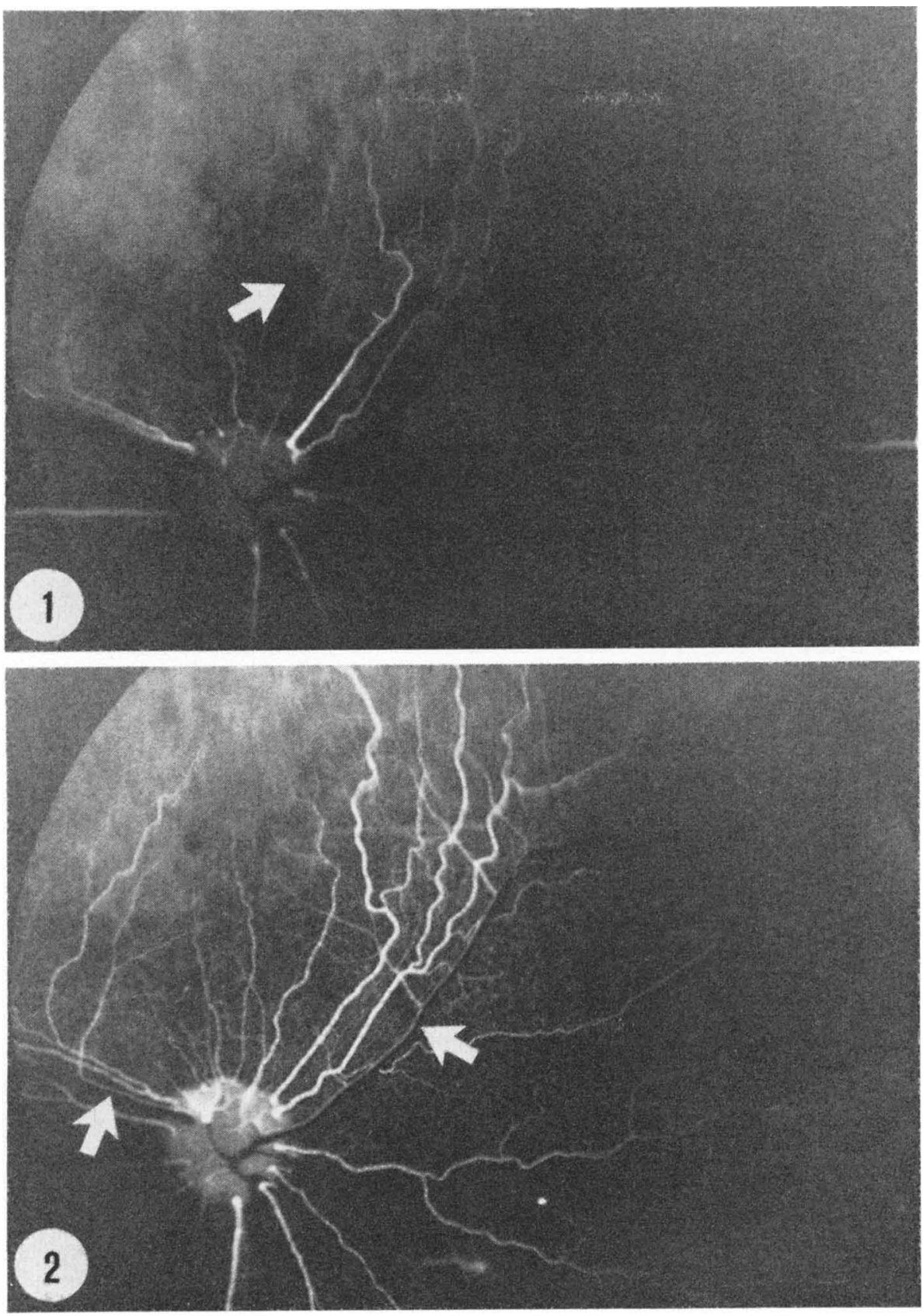

Figure 1. Dog A. The fundus of a 4 year old Miniature Poodle bitch photographed in the early arteriolar phase. Fluorescein shows up in different shades of whithe. Empty vessels appear as black lines. The arterioles are filling at slightly different times. Choriocapillaris has started to fill in a patched pattern (white arrow).

F i g u r e 2. Dog A. Early venous phase. Most of the cilioretinal arterioles are fulorescing. The optic disc shows some fluorescence. Two large vein stems show laminated dyeflow (white arrows). Choriocapillaris fluorescence is more diffuse due to the masking effect of the pigment epithelium. 

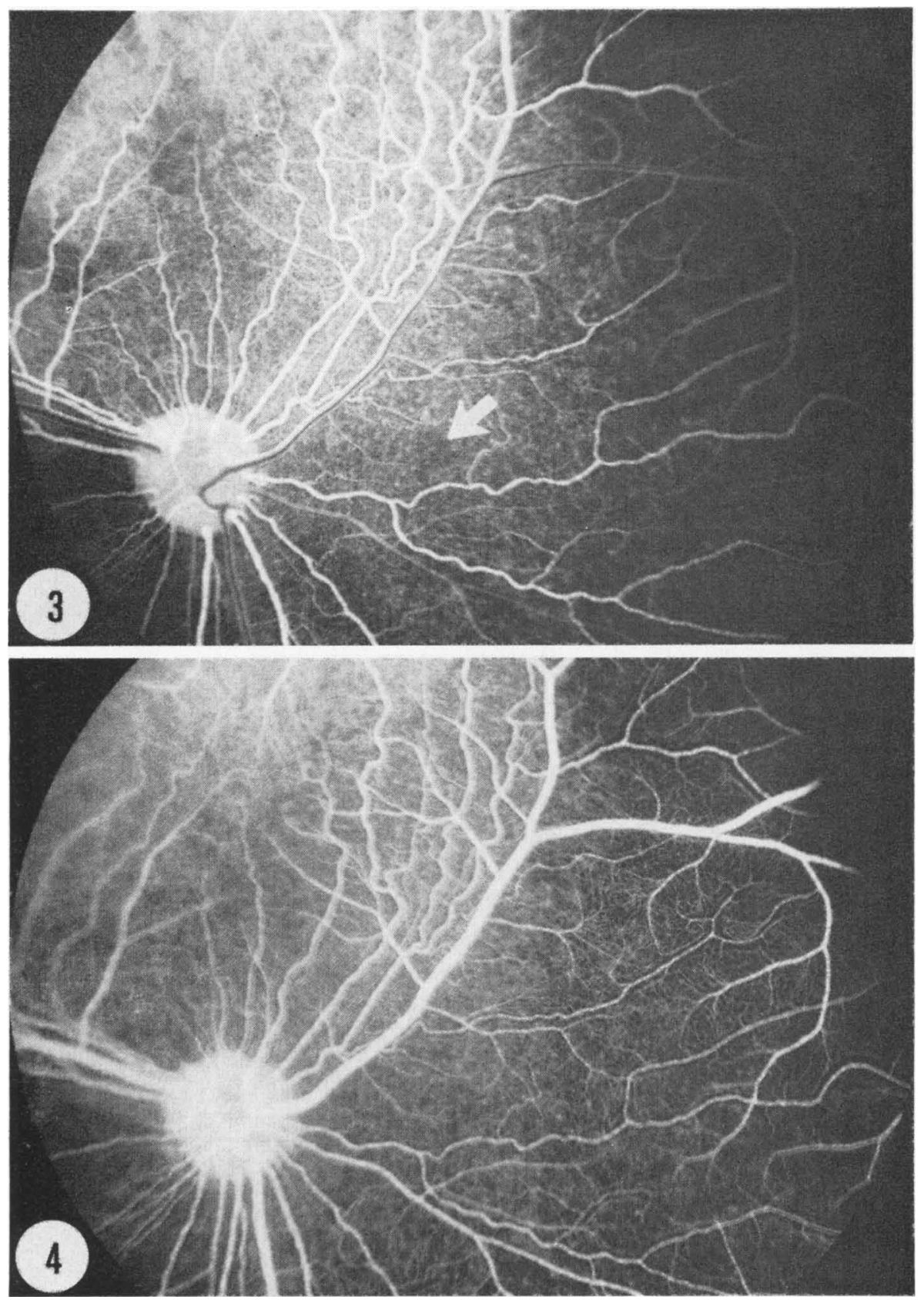

Fig u re 3. Dog A. Mid venous phase. Patches of choriocapillaris layer still remain unfilled (white arrow). The lamination of fluorescein in the large veins is now clearly visible. The arterioles are fluoresceing intensely. The capillaries near the optic disc have started to fill.

Figu re 4. Dog A. Late venous phase. All the veins are filled with dye. The peripherial capillary network shows vivid staining 

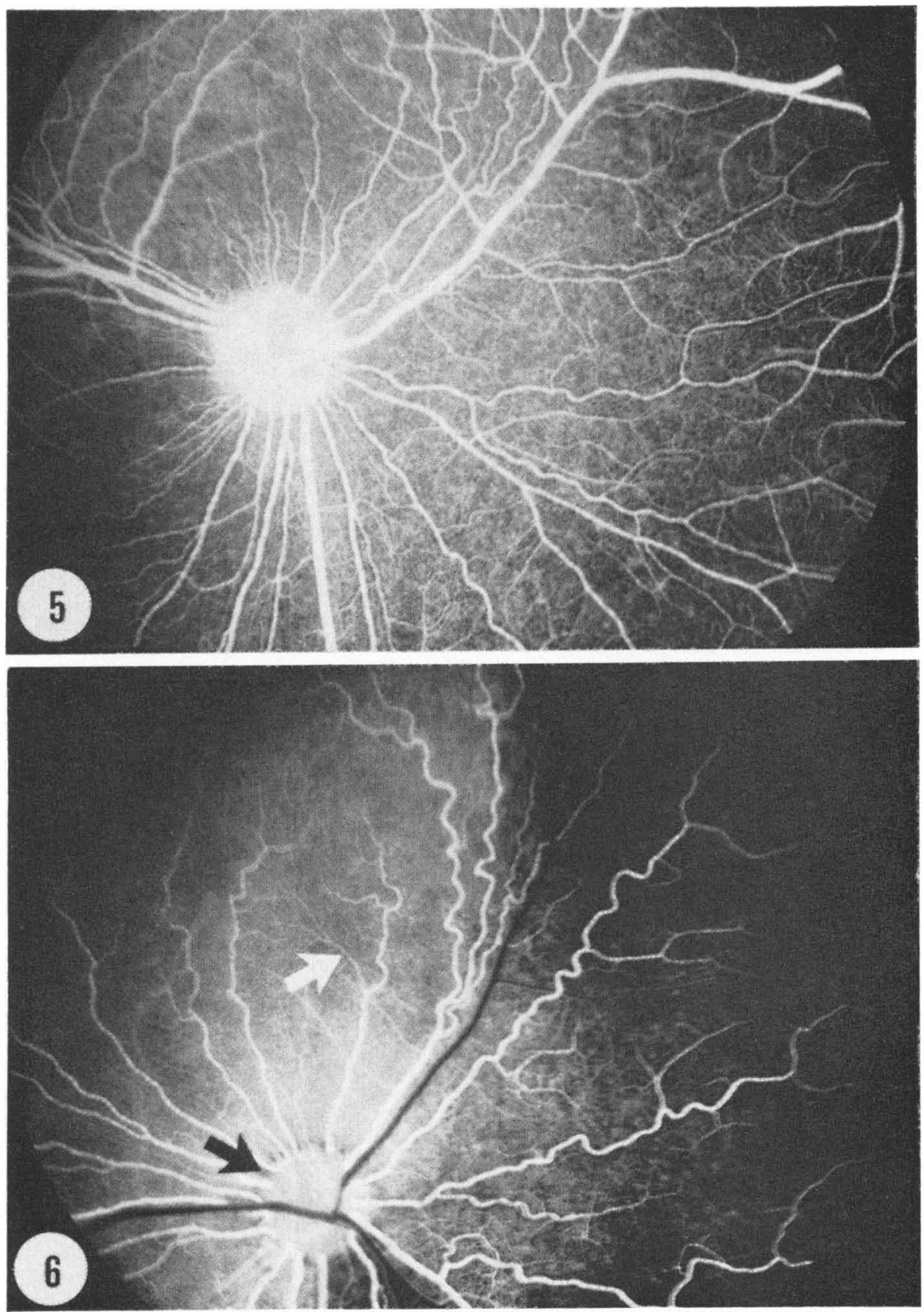

Figure 5. Dog A. Late phase of angiography. No dye leakage is seen.

Figure 6. Dog B. The fundus of a 2 year-old Miniature Poodle bitch photographed in the very early venous phase. The tertiary and secondary branches of the veins from the central area have already filled (white arrow). The optic disc is surrounded by a narrow hypofluorescent ring due to peripapillary pigment aggregation (back arrow). Two kinds of arterioles are fluorescing: 16 long tourtuous cilioretinal vessels reaching throughout the retina, and shorter and thinner arterioles leading to the peripapillary region. 

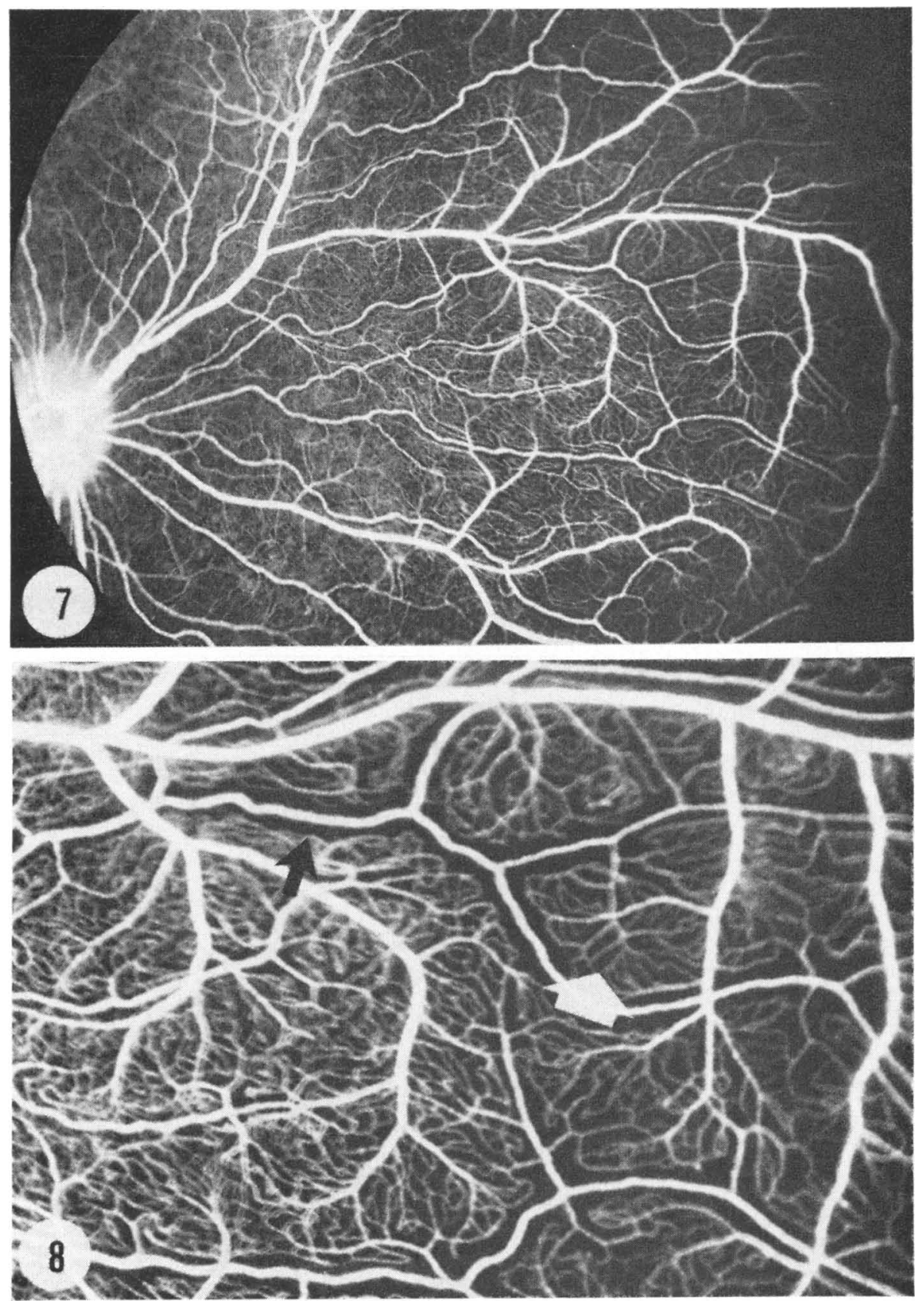

Figure 7. Dog C. The fundus of a 4 year old Toy Poodle bitch photographed in the late venous phase. The decrease in the density of the capillary network towards the periphery is obvious. No arteriovenous shunt formation is seen.

Figu re 8. An enlarged segment from Fig. 7 showing the midperipheral fundus of $\operatorname{dog} \mathrm{C}$. The fine capillary meshwork is clearly visible. The capillaries form freely anastomosing loops. The capillaries are arranged in a lobulated pattern with indistinct margins (white arrow). A single arteriolar branch seems to feed each lobulus which is drained by a small venular branch. A capillary free zone is evident along the arterioles (black arrow). 
vessel walls in major vein stems (Figs. 2 and 3.). This phenomen was not seen in the small venules which were completely filled with dye (Fig. 3). The laminated pattern was not disturbed where 2 veins joined (Fig 3 ). The laminated flow of the dye was maintained all the way to the optic disc (Fig. 3). In the late-venous phase the whole lumen of the larger veins were filled with dye (Fig. 4). No dye leakage from retinal vessels was observed during the whole angiography.

With the use of a combination of ketamine and xylazine for anesthesia the dogs were easily handled and positioned for photography. In most of the dogs the globe remained straight during the whole procedure, which was an advantage of this anesthesia compared to halothane. Only in 2 dogs did the globe slightly rotate ventronasally in the beginning of the angiography, but the globe was easily straightened with a muscle hook. The duration of the anesthesia was $30-40 \mathrm{~min}$, which was obtained with a single dose of anesthetics. For longer duration an extra injection of $4 \mathrm{mg} / \mathrm{kg}$ ketamine was needed. This proved to be unnecessary because most of the dogs remained sufficiently sedated for examination although they showed signs of recovery from anesthesia. In 3 dogs rapid horizontal hystagmus appeared at the beginning of the anesthesia. Three Poodles, 2 Labrador Retrievers and 1 Chesapeake Bay Retriever developed epileptiformic seizures of short duration during the first 5 min after injection of the anesthetics.

\section{Complications}

One 2 year-old Giant Poodle with no previous signs of respiratory or circulatory disease died of pulmonary edema 2 days after angiography; necropsy revealed a myocardial degeneration.

\section{DISCUSSION}

A detailed study of the retinal vessels and the way they filled with fluorescein was possible with the use of a fundus camera with a wide angle lens and Kodak Tri-X pan 400 ASA film. The dosage of $12 \mathrm{mg} / \mathrm{kg}$ sodium fluorescein, which is the dosage used for humans, proved to be adequate for dogs. With this dosage all the different phases of the angiography were well visualized. After injection of the fluorescein side effects such as vomiting were rare. The use of control photographs, taken prior 
to the injection of the dye, allowed for easier topographic orientation when evaluating fundus angiograms. The evidence of tapetal autofluorescence during angiography indicates that the tapetum emits wave-lengths in the same range as fluorescein. The tapetal autofluorescence combined with choroidal fluorescence inhibits the visualization of dye filled capillaries in the tapetal region. The choriocapillaris fluorescence was diffuse due to dye leakage starting from the early venous phase. The capillaries were easily distinguished in dogs with a darkly pigmented fundus. The more clearly defined capillaries in the peripheral fundus could result from a combination of their lesser density and a stronger pigmentation of the pigment epithelium. The avascular zone was angiographically seen to lineate only the retinal arterioles and not the venules. This corresponds to observations made in humans (Nowotny \& Alvis 1960, 1961). The fluorescein filled arterioles and veins seemed larger in diameters when compared to the nonfluorescent vessels in the control photographs. The phenomen has been previously reported in man (Meyer \& Schwickerath 1968). This is explained by the binding of the dye to the peripheral plasmacolumn. Blood plasma is ophthalmoscopically invisible. No specific sex, age or breed differences were observed in our results.

For angiographic procedures the use of a combination of ketamine and xylazine proved to be very useful due to the limited effects on the globe and sufficient duration of sedation. The authors believe that the anesthesia could be a contributory factor to the fatal disorders of 1 Giant Poodle mentioned previously. Therefore ketamine in dogs should be used with caution.

\section{ACKNOWLEDGEMENTS}

The authors highly appreciate the advice and criticism offered by Docent Christina Raitta, M.D., Department of Ophthalmology, University of Helsinki. This study was supported by Silmäsäätiö, The Eye Foundation.

\section{REFERENCES}

Bellhorn, R. W.: Fluorescein fundus photography in veterinary opthalmalogy. J. Amer. Anim. Hosp. Ass. 1973, 9, 227-233.

Gelatt, $K$. N.: Ophthalmologic examination and diagnostic procedures. In: Gelatt, K. N. (ed.) Textbook of Veterinary Ophthalmology. Lea \& Febiger, Philadelphia 1981, p. 206-261. 
Gelatt, K. N., J. P. Henderson \& R Steffen: Fluorescein angiography of the normal and diseased ocular fundi of the laboratory dog. J. Amer. vet. med. Ass. 1976, 169, 980—984.

Nowotny, H.R.\& D. L. Alvis: A method of photographing fluorescence in circulating blood of the human eye. Amer. J. Ophthal. 1960, $50,176$.

Nowotny, H.R.\& D. L. Alvis: A method of photographing fluorescence in circulating blood in the human retina. Circulation 1961, 24, $82-86$.

Wessing, A. \& G. Schwickerath: Fluoreszens-angiographie der Retina. Lehrbuch und Atlas. (Fulorescein angiography of the retina. Textbook and atlas). Georg Thieme Verlag, Stuttgart 1968, p. 41.

\section{SAMMANFATTNING}

Fluorescein angiografi av hundens ögonbotten $i$ ketamin-xylazin anestesi.

Fluorescine fundus angiografi med en Canon $\mathrm{CF} 60 \mathrm{Z}$ vidvinkel kamera gjordes på 30 hundar som icke visade patologiska förändringar i ögonbottnen. I bilder tagna i serie kunde fyra skilda cirkulationsfaser av fluoresceinflödet åtskiljas: den choroidala, näthinnas arteriola, den kapillära och den venösa fasen. Färgämnets genomflöde i näthinnans kärl beskrevs i detalj. Kombinationen av Ketamin och Xylazin visade sig vara lämplig för anestesin.

(Received May 7, 1984).

Reprints may be requested from: B. Kommonen, the Department of Surgery, College of Veterinary Medicine, Hämeentie 57, 00550 Helsinki 55, Finland. 